\title{
Histopatología del Hígado, Lipoperoxidación e Índices Somáticos de Fulica americana en los Humedales de Xochimilco (Urbano) y Tecocomulco (Rural) de la Cuenca de México
}

\author{
Liver Histopatology, Lipidperoxidation and Somatic Indices of Fulica americana \\ in Xochimilco (Urban) and Tecocomulco (Rural) Wetlands in the Mexico Basin
}

María Eugenia López-Islas*; Itzel Ibarra-Meza*; Esperanza Ortiz-Ordóñez*; Liliana Favari**; J. Elías Sedeño-Díaz **** \& Eugenia López-López

LÓPEZ-ISLAS, M. E.; IBARRA-MEZA, I.; ORTIZ-ORDÓÑEZ, E.; FAVARI, L.; SEDEÑO-DÍAZ, J. E. \& LÓPEZ-LÓPEZ, E. Histopatología del hígado, lipoperoxidación e índices somáticos de Fulica americana en los humedales de Xochimilco (urbano) y Tecocomulco (rural), de la Cuenca de México. Int. J. Morphol., 34(2):522-532, 2016.

RESUMEN: Los humedales son cuerpos de agua muy productivos y son refugio para aves migratorias; sin embargo, continuamente reciben contaminantes que ejercen daños en los organismos acuáticos. El objetivo del trabajo fue evaluar el estado de salud de Fulica americana mediante biomarcadores histopatológicos, de estrés oxidativo, e índices somáticos en dos humedales de la Cuenca de México, para evaluar el efecto de las condiciones del hábitat en la salud de las aves. Se obtuvieron ejemplares en los humedales, Xochimilco (X) y Tecocomulco (T) en diferentes períodos de un año. Se determinó su edad, se evisceraron y pesaron, se obtuvieron porciones del hígado para el análisis histológico y para la cuantificación del nivel de lipoperoxidación (LPO). Se analizó la calidad del agua de X y T. Se realizó la técnica histológica de inclusión en parafina y la tinción Hematoxilina-Eosina. El mayor deterioro de la calidad del agua se observó en X. Se encontró infiltración leucocitaria (IN) y vasocongestión (VA) en tres grados. En T se observó la mayor prevalencia de ejemplares con daños combinados, en particular en las hembras; mientras que en X se presentó un mayor porcentaje de organismos con IN. La LPO más alta se observó durante el invierno en T lo que pudiera estar relacionado con el arribo de aves migratorias y la cacería; mientras que en X la mayor LPO estuvo relacionada con la reproducción. La mayor masa corporal eviscerada se presentó en los machos de T y la menor en las hembras de X. En todas las gallaretas los índices hepatosomáticos máximos se alcanzaron en otoño y los mínimos en primavera. F. americana puede ser utilizada como centinela debido a que presentó respuestas diferenciadas en los índices somáticos y en los biomarcadores histopatológicos y de estrés oxidativo en las distintas localidades, sexo y épocas del año.

PALABRAS CLAVE: Ave acuática; Biomarcadores histológicos; Estrés oxidativo; Humedales Ramsar; Variación estacional.

\section{INTRODUCCIÓN}

Los humedales son ecosistemas muy productivos que albergan una gran biodiversidad, especialmente aves acuáticas. En las últimas décadas han disminuido drásticamente y han sido objeto de una continua degradación por el desarrollo de infraestructuras, la conversión de tierras, la extracción de agua, la eutrofización, la contaminación y la introducción de especies exóticas, lo cual afecta negativamente a las poblaciones de aves acuáticas residentes y migratorias ya que constituyen sus áreas de reproducción, alimentación, refugio y descanso (Ramsar, 2015).
Los biomarcadores son una herramienta muy útil para evaluar la salud de los organismos acuáticos ya que permiten detectar daños tempranos resultado de la exposición a diversos contaminantes. En muestras de campo, los biomarcadores pueden ser indicadores de la exposición a la carga total de los compuestos tóxicos biodisponibles en los ecosistemas naturales, entre los más usados están los biomarcadores de estrés oxidativo y los histopatológicos (van der Oost et al., 2003).

\footnotetext{
* Laboratorio de Evaluación de la Salud de los Ecosistemas Acuáticos. Escuela Nacional de Ciencias Biológicas, Instituto Politécnico Nacional, México D.F., México.

** Departamento de Farmacología, Centro de Investigación y de Estudios Avanzados, Instituto Politécnico Nacional, México D.F., México.

*** Coordinación Politécnica para la Sustentabilidad, Instituto Politécnico Nacional, México D.F., México.

El financiamiento para el desarrollo del presente trabajo fue otorgado por la Secretaría de Investigación y Posgrado del Instituto Politécnico Nacional, con números de registro: SIP 20111126, 20110602 y 20120931.
} 
LÓPEZ-ISLAS, M. E.; IBARRA-MEZA, I.; ORTIZ-ORDÓÑEZ, E.; FAVARI, L.; SEDEÑO-DÍAZ, J. E. \& LÓPEZ-LÓPEZ, E. Histopatología del hígado, lipoperoxidación e índices somáticos de Fulica americana en los humedales de Xochimilco (urbano) y Tecocomulco (rural), de la Cuenca de México. Int. J. Morphol., 34(2):522-532, 2016.

El estrés oxidativo es el desequilibrio entre prooxidantes y antioxidantes a favor de los oxidantes, que potencialmente pueden causar daños oxidativos al ADN, las proteínas y lípidos. Entre las principales moléculas "blanco" de las especies reactivas de oxígeno, se encuentran los lípidos de las membranas celulares, las cuales al ser dañadas por el hidroxilo provocan la lipoperoxidación (LPO). Una LPO extensiva ha sido correlacionada con la ruptura de la membrana y la muerte celular, lo que provoca daños tisulares y orgánicos que afectan la salud del organismo (Koivula \& Eeva, 2010). Cuantificar el estrés oxidativo permite identificar daños tempranos, con lo cual es posible anticipar cambios en diferentes niveles de organización: molecular, celular, histológico, individual, población, comunidad y ecosistema, permitiendo la aplicación de medidas correctivas antes de que ocurra un daño ambiental irreversible. En aves se han observado diversos daños histopatológicos ocasionados por la LPO, tales como congestión hepática, vacuolización, degeneración granular e infiltración por linfocitos (van der Oost et al.).

El hígado es considerado uno de los órganos de gran importancia fisiológica, ya que realiza numerosas funciones como son: el almacenamiento y la biotransformación de sustancias recibidas a través del torrente sanguíneo; la detoxificación; la síntesis y el almacenamiento de glucógeno; la producción de glucosa y la gluconeogénesis durante la escasez de nutrientes, vertiendo al torrente sanguíneo la glucosa necesaria para todos los tejidos. Además, juega un papel importante en el periodo de reproducción de las aves, durante la vitelogénesis y el crecimiento de los oocitos, porque sintetiza la vitelogenina y las lipoproteínas, las cuales son las fuentes principales de proteínas y lípidos, respectivamente, proveyendo los nutrientes y la energía que requiere el desarrollo del embrión (Williams, 2012). Por lo cual es un órgano muy vulnerable a la exposición de diferentes tóxicos.

Una de las limitaciones al utilizar biomarcadores de estrés oxidativo en estudios ambientales en aves, es la escasez de información acerca de las variaciones estacionales y de los niveles basales en sangre o en tejidos (Raja-aho et al., 2012). En la fauna silvestre, los ciclos anuales están sincronizados con las variaciones temporales del ambiente (fotoperiodo, temperatura, época de lluvia y de sequía y disponibilidad de alimento), afectando la morfología, la fisiología y el comportamiento de los organismos (Wikelski et al., 2008).

En las especies de aves no migratorias la reproducción y la muda son las etapas cruciales del ciclo anual el cual incluye: la muda postnupcial; un cierto grado de almacenamiento de grasa invernal, la muda prenupcial, el crecimiento gonadal y las actividades de reproducción, cada una de ellas con altas demandas energéticas, por lo que son actividades que tienen lugar a lo largo del año y el traslape entre ellas es mínimo (Williams).

Por otro lado, los índices somáticos, como la masa corporal eviscerada (MCE), el índice hepatosomático (IHS) y el gonadosomático (IGS), permiten identificar la condición biológica de los organismos. Estos índices han sido utilizados como características importantes de los organismos en la evaluación de la sobrevivencia, la reproducción y el comportamiento (Labocha \& Hayes, 2012). El uso combinado de biomarcadores de daño temprano e índices somáticos constituye una mejor aproximación para la evaluación de la salud de los organismos silvestres.

El objetivo del presente trabajo fue evaluar el estado de salud del ave acuática Fulica americana, de un humedal urbano y otro rural de la Cuenca de México, analizando el nivel de LPO, los daños tisulares en hígado e índices somáticos (MCE, IHS e IGS), durante un ciclo anual, considerando las variaciones de los factores fisicoquímicos del agua de ambos humedales, para identificar el efecto de las condiciones ambientales de los humedales sobre la salud de F. americana.

\section{MATERIAL Y MÉTODO}

Área de estudio. Los humedales de Tecocomulco y Xochimilco se ubican en la Cuenca de México (Fig. 1A). Xochimilco (Fig. 1B), se encuentra en la Ciudad de México, una de las urbes más pobladas del mundo, se mantiene con aguas residuales tratadas y recibe descargas de drenaje doméstico. Es un humedal heterogéneo con zonas contrastantes, la urbana, la turística y la chinampera. Tecocomulco (Fig. 1C), es una zona rural considerada un relicto ecológico de la biodiversidad de los lagos de la Cuenca de México (Ramsar), dominado por ocho especies de macrófitas. Ambos humedales reciben aportes de nutrientes por las actividades agrícolas que se efectúan en sus alrededores. Además, son el hábitat de una gran variedad de especies de flora y fauna, de manera que constituyen áreas de descanso y de refugio de las aves migratorias, por lo que son considerados Humedales de Importancia Internacional Ramsar. En Tecocomulco, se han registrado 42 especies de aves acuáticas migratorias (que arriban en grandes parvadas de octubre a marzo) y siete residentes; y en Xochimilco, 47 especies de aves acuáticas migratorias (con parvadas menores que las de Tecocomulco) y 7 residentes (Ramsar).

Especie estudiada. Fulica americana (gallareta), es una especie de ave acuática muy abundante y de amplia distribu- 

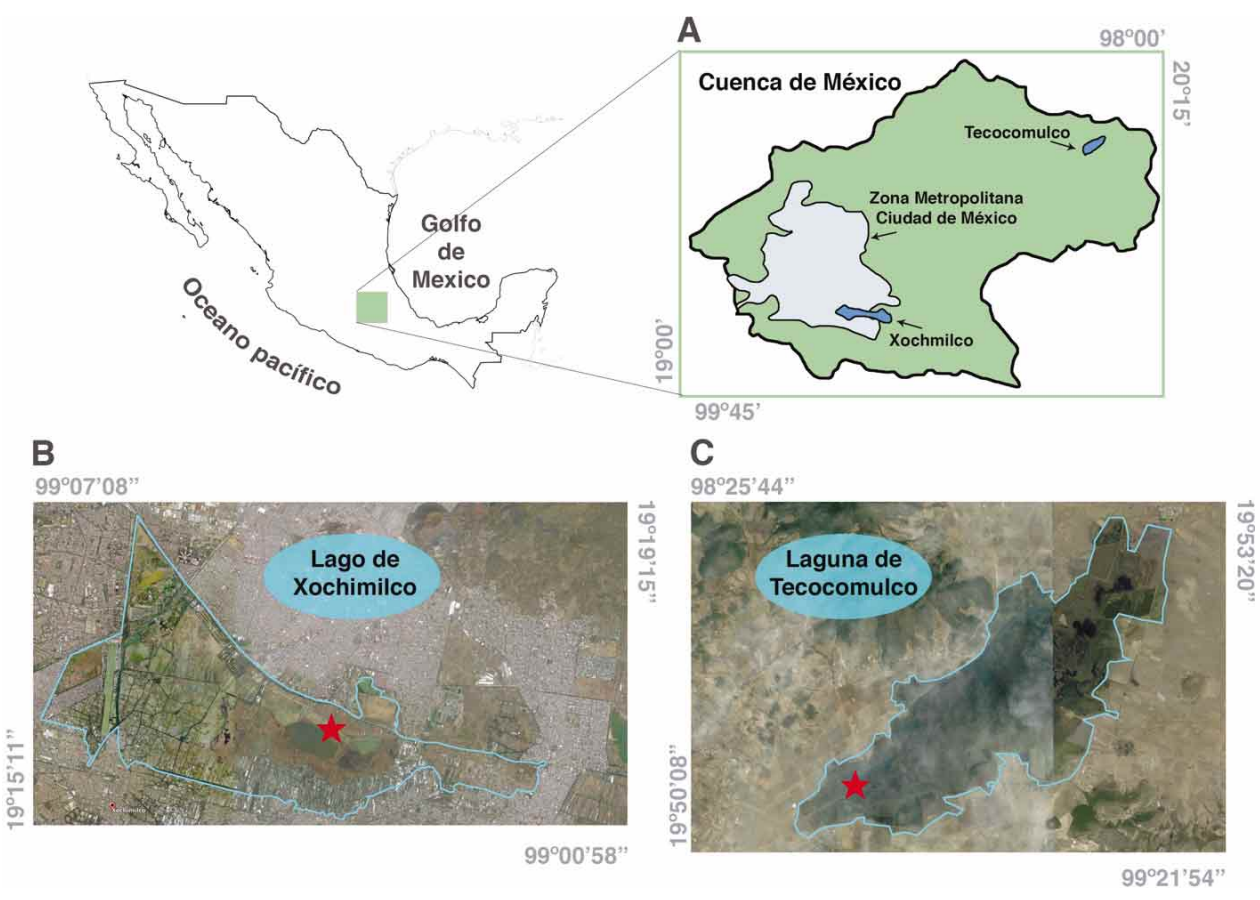

Fig. 1. Mapa de ubicación de las áreas de estudio de las poblaciones de Fulica americana: A) La Cuenca de México, B) Lago de Xochimilco y C) Laguna de Tecocomulco. «= Sitios de colecta.

ción en Norteamérica. En México, habita en humedales continentales y costeros donde se encuentran poblaciones tanto residentes como migratorias (Pérez-Arteaga \& Gaston, 2004). Pertenece al orden Gruiformes y a la familia Rallidae, no presenta dimorfismo sexual, es omnívora, se alimenta principalmente de plantas vasculares acuáticas y algas; en la época de reproducción consume insectos, moluscos, crustáceos, anfibios y peces; todo su ciclo de vida lo realiza en el cuerpo de agua (Brisbin et al., 2002). F. americana presenta poblaciones residentes en Tecocomulco y en Xochimilco por lo que se eligió como especie centinela.

Trabajo de Campo. Se realizaron cuatro campañas de trabajo: primavera, verano, otoño e invierno del ciclo anual 2011-2012. Los ejemplares colectados (permiso de la SEMARNAT No. FAUT-0267), fueron eviscerados y pesados, se obtuvieron porciones del hígado para el análisis histológico y el tejido restante se almacenó en nitrógeno líquido para cuantificar la LPO. La edad de las gallaretas se determinó siguiendo los criterios de Pyle (2008). Se analizaron 35 ejemplares de $F$. americana de Tecocomulco y 24 de Xochimilco.

Se registraron diversos factores ambientales: temperatura del agua $\left({ }^{\circ} \mathrm{C}\right), \mathrm{pH}$, oxígeno disuelto $(\mathrm{mg} / \mathrm{L})$, conductividad $(\mu \mathrm{S} / \mathrm{cm})$ y se tomaron muestras de agua que fueron transportadas al laboratorio en obscuridad y refrigeración. Para evaluar la calidad del agua, en el laboratorio se determinaron la demanda bioquímica de oxígeno $\left(\mathrm{DBO}_{5}\right.$; $\left.\mathrm{mgL}^{-1}\right)$, los cloruros $\left(\mathrm{mgL}^{-1}\right)$, la dureza y la alcalinidad (mgL $\mathrm{m}^{-}$ $\left.{ }^{1}\right)$ por titulación y la cuantificación de los coliformes totales y fecales por el método del número más probable (NMP/ $100 \mathrm{~mL}$ ) siguiendo las técnicas estándar de la American Public Health Association (2005). Las concentraciones de $\mathrm{N}$ total $\left(\mathrm{mgL}^{-1}\right)$, nitritos $\left(\mathrm{mgL}^{-1}\right)$, nitratos $\left(\mathrm{mgL}^{-1}\right)$, amoníaco $\left(\mathrm{mgL}^{-1}\right), \mathrm{P}$ total $\left(\mathrm{mgL}^{-1}\right)$, ortofosfatos $\left(\mathrm{mgL}^{-1}\right)$, sulfatos $\left(\mathrm{mgL}^{-}\right.$ ${ }^{1}$ ), sólidos suspendidos totales (SST) $\left(\mathrm{mgL}^{-1}\right)$ y color (unidades Pt - Co) se midieron utilizando un espectrofotómetro Hach DRL 2500.

En el estudio histopatológico del hígado se utilizó la técnica histológica de inclusión en parafina descrita por Uría \& Mora (1996), se realizaron cortes de siete $\mu \mathrm{m}$ con un micrótomo de parafina MICROM HM325, posteriormente se realizaron las tinciones con la técnica de HematoxilinaEosina, y las laminillas obtenidas se analizaron y fotografiaron con un fotomicroscopio Leica ATC 2000. Se obtuvieron los niveles de LPO por la técnica de Buege \& Aust (1978), cuantificando espectrofotométricamente el malondialdehído (MDA) resultante de la LPO, los resultados se expresaron como nmol de MDA mg-1 prot. Las proteínas se determinaron por el método de Bradford (1976). Todos los ensayos fueron realizados por triplicado.

Se obtuvieron los índices somáticos (IS): índice hepatosomático (IHS), índice gonadosomático (IGS) e ín- 


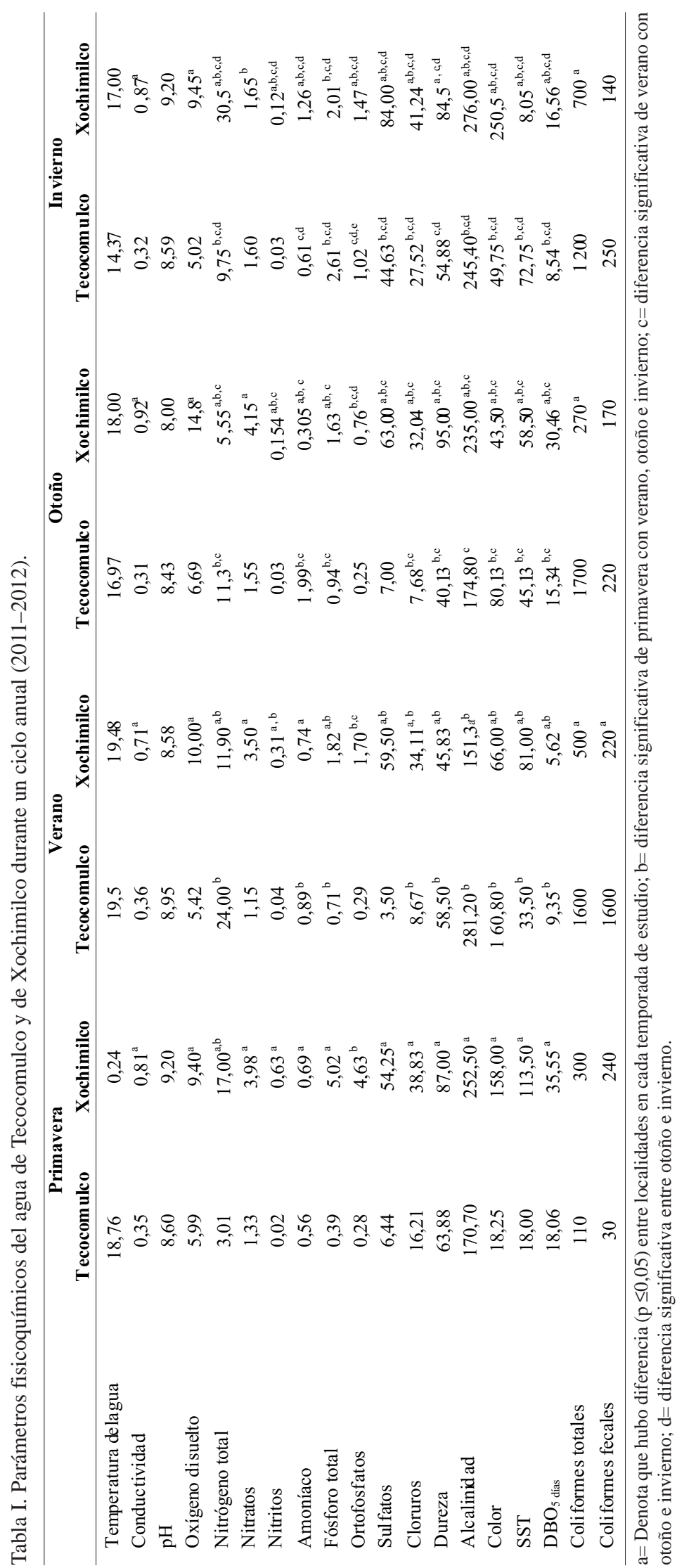

dice de masa corporal eviscerada (MCE), de acuerdo con las relaciones propuestas por Malik et al. (2012) y Labocha \& Hayes (2012).

Se analizó la relación de cada humedal con los parámetros fisicoquímicos mediante un análisis de ordenación de componentes principales (ACP). Se analizaron las diferencias significativas para LPO y los IS, con las pruebas no paramétricas de Kruskal-Wallis y Mann-Whitney ( $p<0,05$ ). Se obtuvieron las prevalencias de las patologías identificadas. Se realizó un análisis de discriminantes y de correlación de Pearson para analizar las relaciones entre la histopatología, la LPO, los IS y la edad.

\section{RESULTADOS}

Características fisicoquímicas del agua. Se encontraron diferencias notables entre las características fisicoquímicas de los dos humedales. Xochimilco alcanzó los valores más altos $(\mathrm{p} \leq 0,05)$ de conductividad, oxígeno disuelto, nitritos, ortofosfatos, sulfatos y cloruros durante las cuatro estaciones del año (Tabla I). Las variaciones estacionales mostraron que durante la primavera en Xochimilco, se alcanzaron los valores máximos de nitritos, $\mathrm{P}$ total, ortofosfatos, $\mathrm{SST}_{\text {y }} \mathrm{DBO}_{5}$; en otoño se detectaron los valores máximos de conductividad, oxígeno disuelto, nitratos y dureza; durante el invierno se registraron los máximos de $\mathrm{N}$ total, sulfatos, cloruros y color. En Tecocomulco, en el verano se alcanzaron los niveles máximos de alcalinidad y de coliformes fecales; y durante el otoño de amoníaco y de coliformes totales (Tabla I).

El ACP (Fig. 2) mostró en sus dos primeros componentes el $61,03 \%$ de la varianza explicada (36,73\% en el primer componente y 24,31 $\%$ en el segundo componente). El diagrama muestra un gradiente de las condiciones ambientales en el que se ubican dos grupos, el de Tecocomulco en los cuadrantes del margen izquierdo y el de Xochimilco en los del margen derecho. En el margen derecho se agruparon los sitios con los valores más altos de $\mathrm{P}$ total, ortofosfatos, temperatura del agua, nitritos, cloruros, conductividad, SST y sulfatos, caracterizando a la primavera y al verano de Xochimilco; el otoño se separó hacia el cuadrante inferior derecho asociado a los valores al- 
tos de la $\mathrm{DBO}_{5}$, la dureza, los nitratos y el oxígeno disuelto; el invierno de Xochimilco se localizó en el cuadrante superior derecho caracterizado por los valores altos del color, la alcalinidad, el $\mathrm{N}$ total y el $\mathrm{pH}$. El verano, el otoño y el invierno de Tecocomulco ubicados en el cuadrante superior izquierdo se caracterizan por presentar altos valores del amoníaco, coliformes totales y fecales; la primavera de Tecocomulco se situó en el cuadrante inferior izquierdo distinguiéndose por presentar los valores más bajos de color, $\mathrm{N}$ total, nitritos, $\mathrm{P}$ total, coliformes totales y fecales (Tabla I y Fig. 2).

Índices somáticos. Los mayores niveles del IGS de hembras y machos se presentaron en primavera y verano, lo que muestra que la reproducción se presenta durante este periodo en ambas localidades (Fig. 3A). Sin embargo, las hembras de Xochimilco presentaron un IGS menor que las hembras de Tecocomulco (Fig. 3A).

Los valores de la MCE de los machos de ambos sitios de estudio fueron mayores $(\mathrm{p} \leq 0,05)$ a los de las hembras (Fig. 3B). En Xochimilco no se detectaron diferencias estacionales de la MCE ( $p>0,05)$. Sin embargo, en Tecocomulco, la MCE de las hembras en primavera y en otoño fue mayor $(\mathrm{p} \leq 0,05)$ a la de las hembras en invierno. Los machos de Tecocomulco durante la primavera presen- taron una MCE mayor ( $\mathrm{p} \leq 0,05)$ que en verano. Además, la MCE de las hembras de Xochimilco de primavera y verano fue menor $(\mathrm{p} \leq 0,05)$ a la de las hembras de Tecocomulco en verano y otoño. Los machos de Tecocomulco presentaron una MCE mayor en la primavera que la de los machos de Xochimilco en el verano ( $<<0,05)$ (Fig. 3B).

Por su parte, el patrón de variación estacional del IHS fue diferente en las hembras y los machos de ambos humedales, las hembras de Xochimilco alcanzaron el mayor valor del IHS durante el verano y el otoño ( $p \leq 0,05$ ); mientras que las hembras de Tecocomulco tuvieron un IHS mayor en otoño e invierno ( $\mathrm{p} \leq 0,05$ ), el IHS menor se observó en la primavera en ambas localidades (Fig. 3C). Los machos de Xochimilco tuvieron un menor IHS en primavera y verano alcanzando el máximo valor durante el otoño ( $\mathrm{p}$ $\leq 0,05)$. Los machos de Tecocomulco no presentaron diferencias significativas en el IHS entre las distintas épocas del año. El IHS de los machos en Xochimilco durante el otoño, fue mayor $(\mathrm{p} \leq 0,05)$ que el de los machos de Tecocomulco durante la primavera y el invierno (Fig. 3C).

Nivel de Lipoperoxidación. Los machos y las hembras de Xochimilco registraron un patrón estacional similar en los niveles de LPO, ya que tuvieron los valores más bajos ( $\mathrm{p}$ $\leq 0,05$ ) durante la primavera y el otoño (Fig. 3D); las hem-

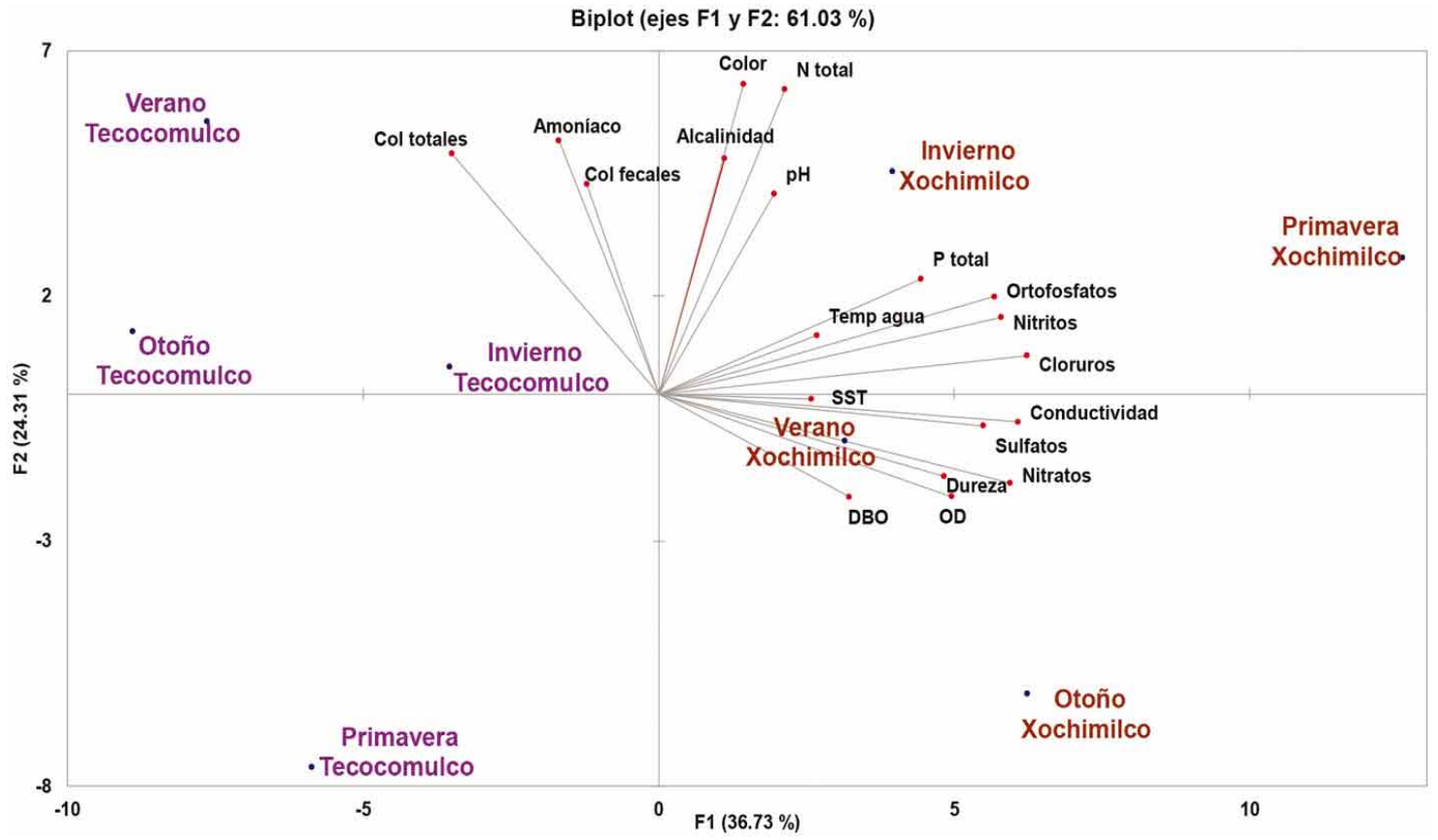

Fig. 2. Diagrama de ordenación de los sitios y temporadas de estudio de acuerdo con sus factores ambientales, durante las cuatro estaciones de estudio. Temperatura del agua $\left({ }^{\circ} \mathrm{C}\right)$, conductividad $(\mathrm{mScm}-1)$, DBO5 días $(\mathrm{mgL}-1)$, cloruros (mgL-1), dureza (mgL-1), alcalinidad (mgL-1), $\mathrm{N}$ total (mgL-1), nitritos (mgL-1), nitratos (mgL-1), amoníaco (mgL-1), P total (mgL-1), ortofosfatos (mgL-1), sulfatos (mgL-1), sólidos suspendidos totales (SST) (mgL-1) y color (unidades Pt - Co), coliformes totales y fecales (NMP/100mL). 

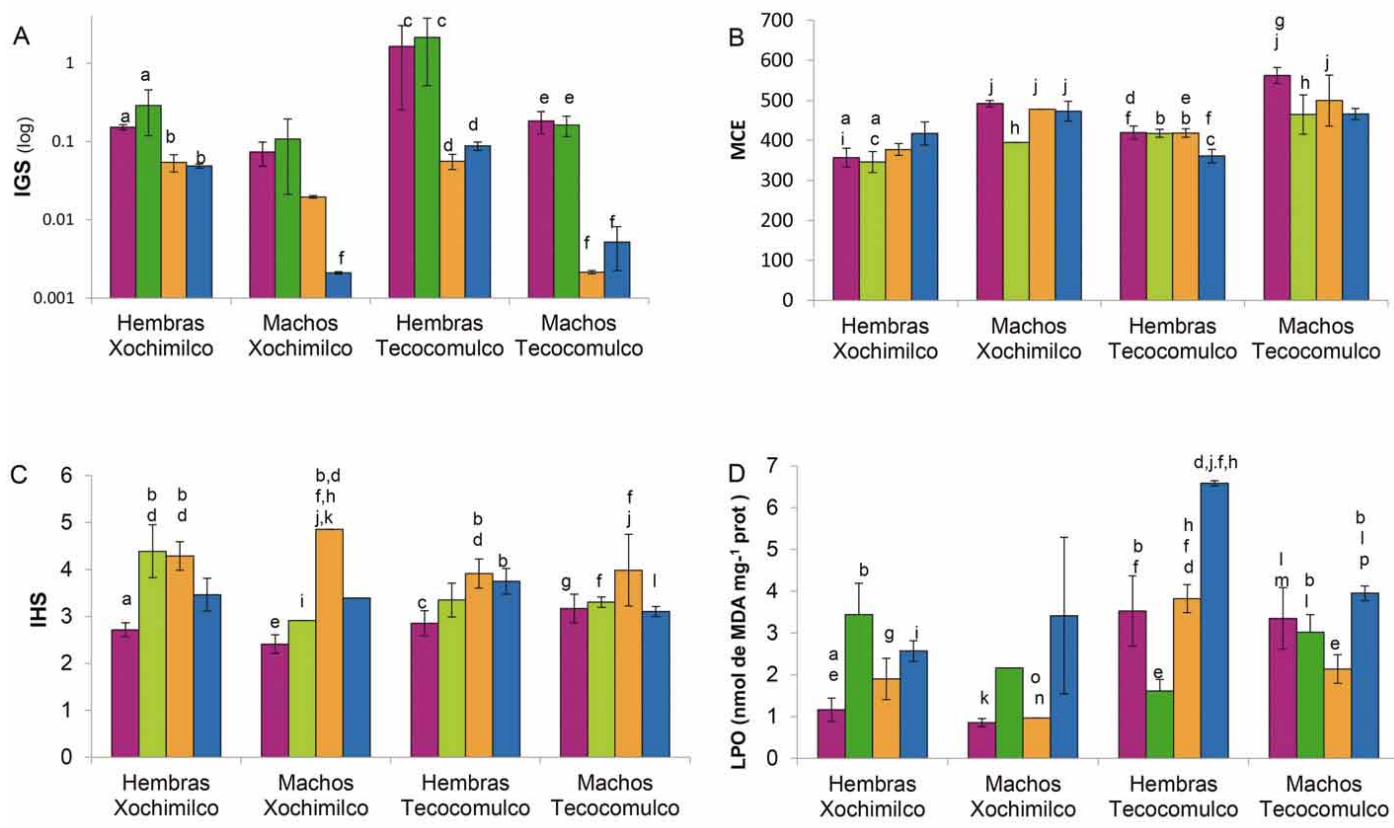

\section{घPrimavera $\quad \square$ Verano $\quad \square$ Otoño $\quad \square$ Invierno}

Fig. 3. Índices somáticos y nivel de lipoperoxidación (LPO) de Fulica americana. A) Índice gonadosomático (IGS), B) Masa corporal eviscerada (MCE), C) Índice hepatosomático (IHS), D) Nivel de lipoperoxidación (LPO).

bras de Xochimilco aumentaron drásticamente sus niveles de LPO durante el verano ( $\mathrm{p} \leq 0,05$ ); los machos de Xochimilco alcanzaron su nivel más alto durante el invierno ( $\mathrm{p} \leq 0,05)$ (Fig. 3D). En contraste, en Tecocomulco las hembras presentaron el menor nivel de LPO en verano y el máximo durante el invierno ( $\mathrm{p} \leq 0,05)$; y los machos de Tecocomulco tuvieron los menores niveles de LPO durante el otoño y los mayores en el invierno (Fig. 3D). Comparando entre humedales, las hembras y machos de Tecocomulco presentaron los niveles de LPO más elevados que las hembras y machos de Xochimilco ( $\leq \leq 0,05)$. El mayor nivel de LPO ( $\leq 0,05)$ fue registrado en las hembras de Tecocomulco durante el invierno. Los machos de Tecocomulco no presentaron variación significativa en el nivel de LPO durante todo el ciclo anual (Fig. 3D).

Estudio Histopatológico. En las gallaretas de ambos sitios de estudio se encontraron en el hígado cambios histopatológicos que corresponden a patrones de reacción como son: alteraciones circulatorias, particularmente la vasocongestión (VA); y la infiltración leucocitaria (IN) en tres grados de intensidad (I, II y III) (Fig. 4). En el hígado de las hembras de Xochimilco en primavera se presentó IN I y II, y en verano IN I, II, III mientras que las hembras de Tecocomulco presentaron durante la primavera VA I y III, aunados a daños combinados de IN y VA (Figs. 5A y 5C). En otoño y en invierno las hembras de Xochimilco y
Tecocomulco presentaron IN y VA en distintos grados y daños combinados (Figs. 5A y 5C). Durante la primavera los machos de Tecocomulco presentaron IN I e IN II y los de Xochimilco IN I e IN III (Figs. 5B y 5D). Los machos de Tecocomulco durante el verano presentaron IN I, IN III y la combinación de IN II-VA III, y los de Xochimilco IN III. Los machos de Xochimilco y de Tecocomulco durante el otoño presentaron distintas combinaciones de IN y VA en diferentes grados (Figs. 5C y 5D). Durante el invierno los machos de Xochimilco sólo presentaron IN y los de Tecocomulco IN y una combinación de ambos tipos de daños (Figs. 5C y 5D).

Relación de los factores ambientales, índices somáticos, nivel de lipoperoxidación, histopatología y edad. El diagrama del Análisis Discriminante de Xochimilco (Figs. 6A y 6B) muestra el 91,20\% de la varianza explicada en sus dos primeros componentes $(74,29 \%$ en el primer componente y $16,91 \%$ en el segundo componente). Se observó que las hembras durante la primavera y el verano se separan de los demás grupos en los cuadrantes inferior y superior izquierdos relacionados con los IGS más altos, son hembras de un año de edad, con IN I y II; las hembras de verano se asocian con los niveles más elevados de LPO. Los machos de primavera y verano se agrupan en el cuadrante inferior derecho junto a los machos de invierno, correlacionados con la mayor MCE, la edad (organismos de 


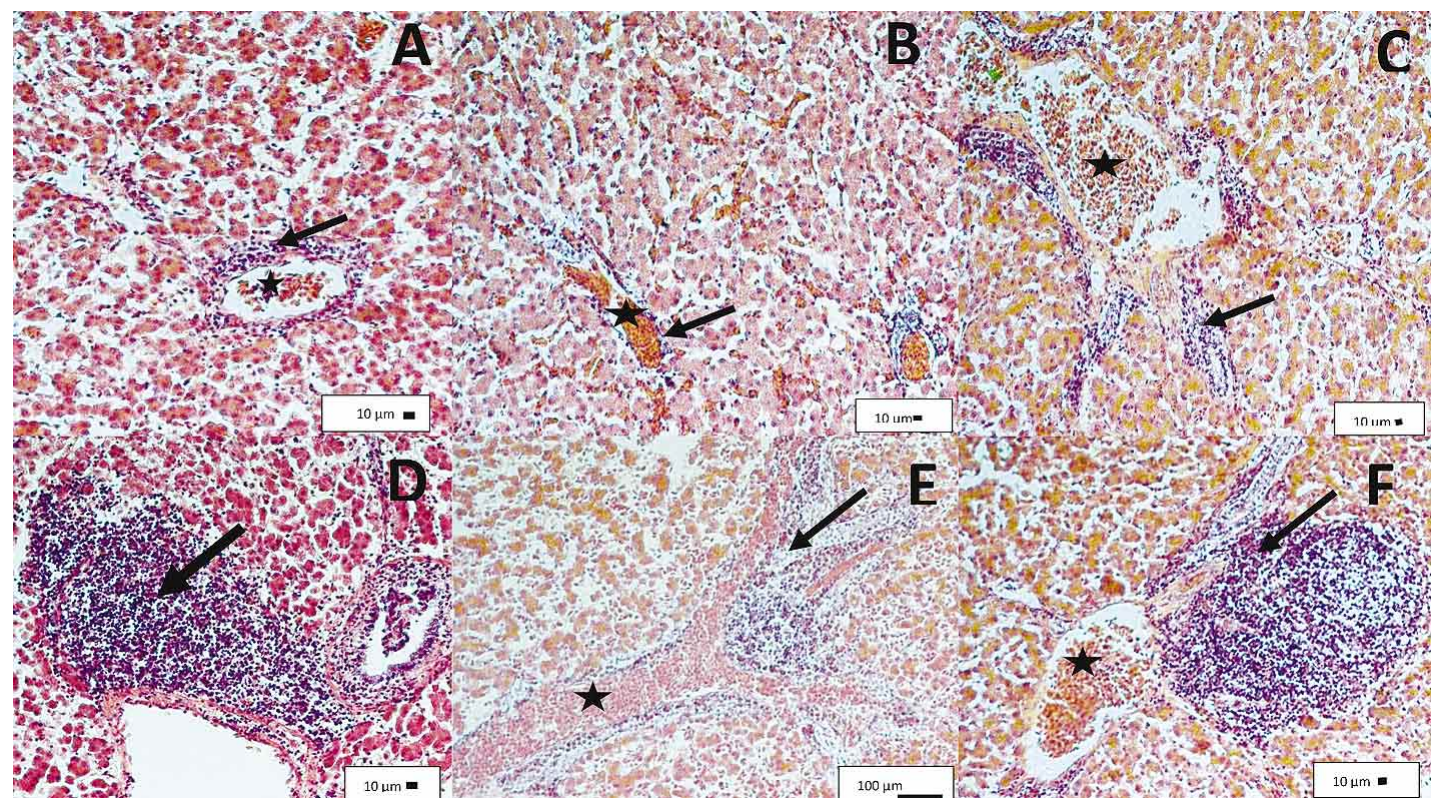

Fig. 4. Daños histológicos observados en F. americana de los humedales de Tecocomulco y Xochimilco: Infiltración leucocitaria IN y vasocongestión (VA) en los grados I, II y III. A) IN-I (flecha). B) IN-I (flecha) y VA-II (estrella). C) IN-II (flecha) VA-II (estrella). D) IN-III (flecha). E) IN-II y VA-III (estrella). F) IN-III (flecha) y VAIII (estrella).

A

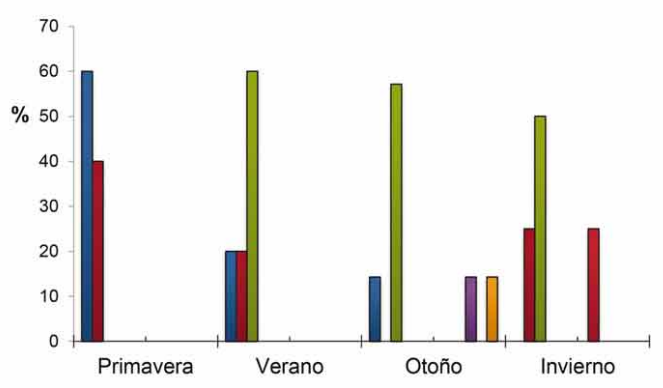

C

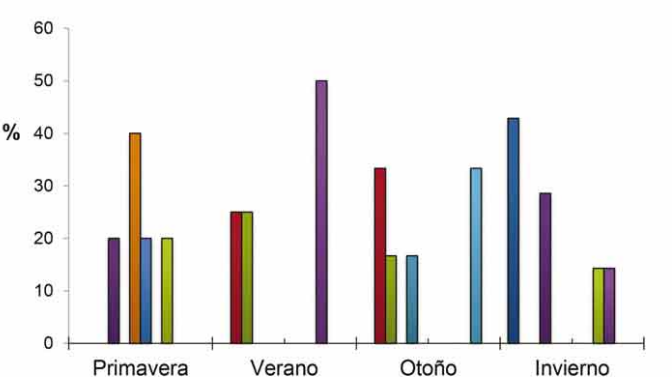

B

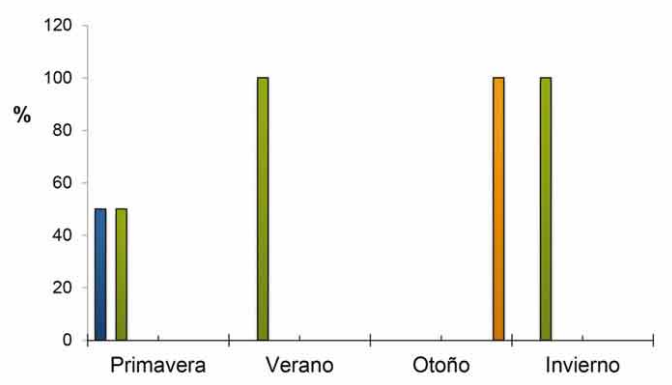

D

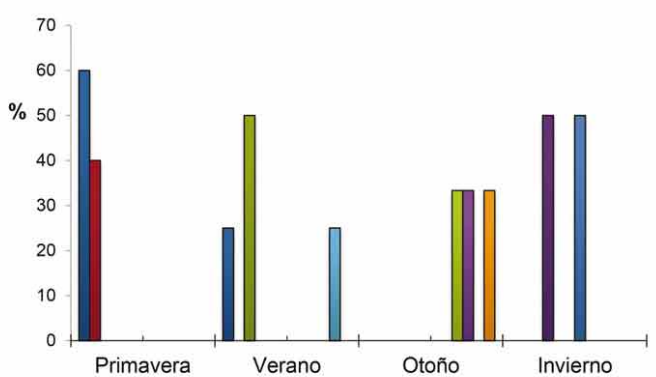

$\square I N I \quad \square I N I I \quad \square I N I I I \quad \square$ VAI $\square$ VAII $\square$ VAIII

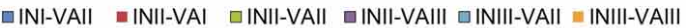

Fig. 5. Porcentajes de las clases de daños histológicos encontrados en el hígado Fulica americana durante el ciclo anual 2011-2012. Simbología: infiltración leucocitaria (IN), vasocongestión (VA), grados de daño I, II, III. A) Hembras de Xochimilco, B) Machos de Xochimilco, C) Hembras de Tecocomulco, D) Machos de Tecocomulco. 
A

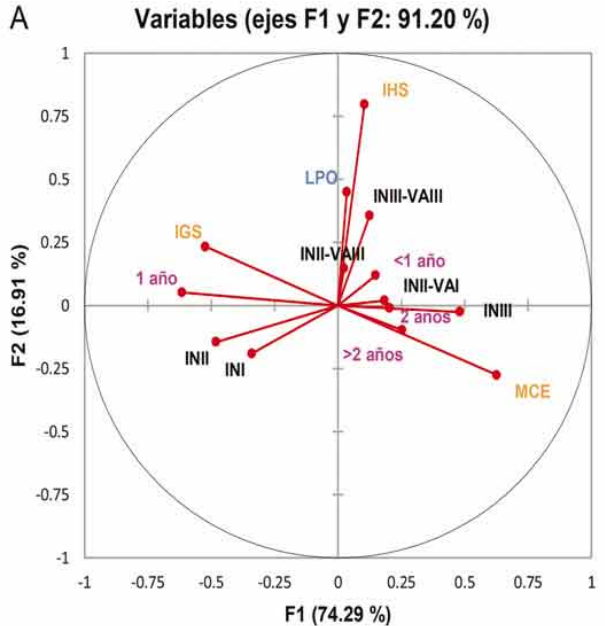

C Variables (ejes F1 y F2: $87.76 \%$ )

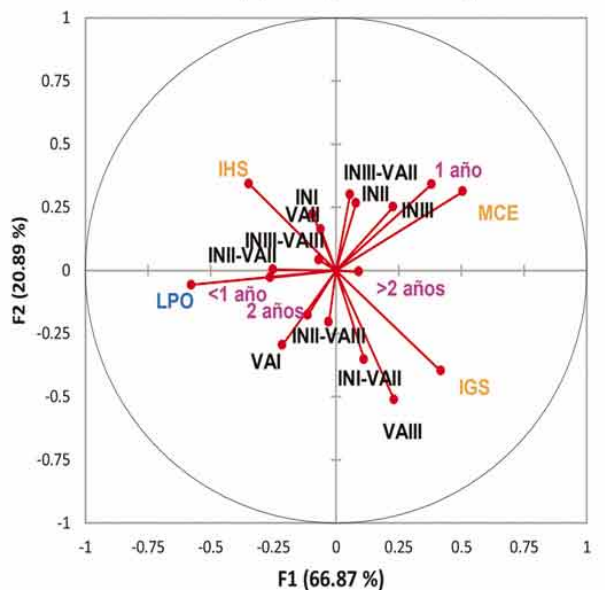

B Centroides (ejes F1 y F2: $91.20 \%$ ) Xochimilco

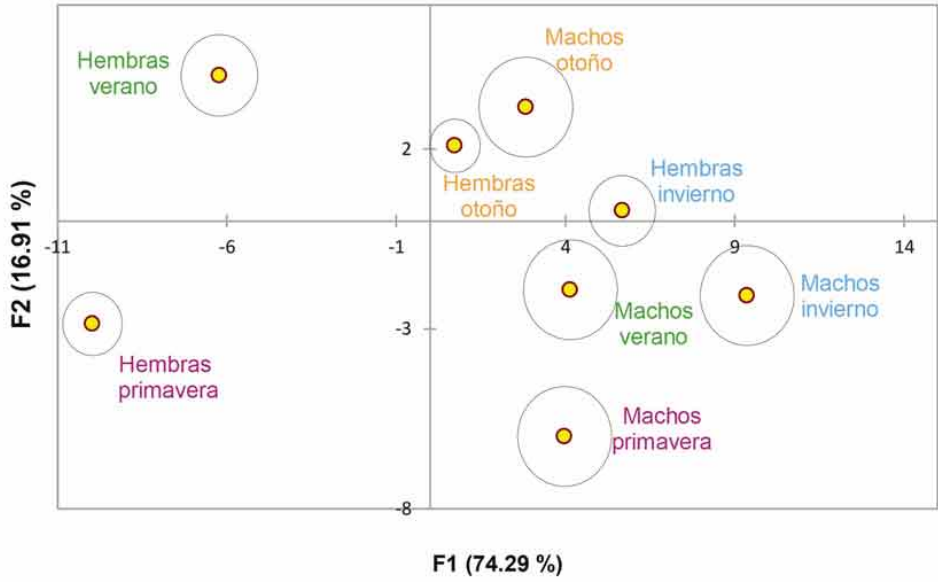

D Centroides (ejes F1 y F2: $87.76 \%$ ) Tecocomulco

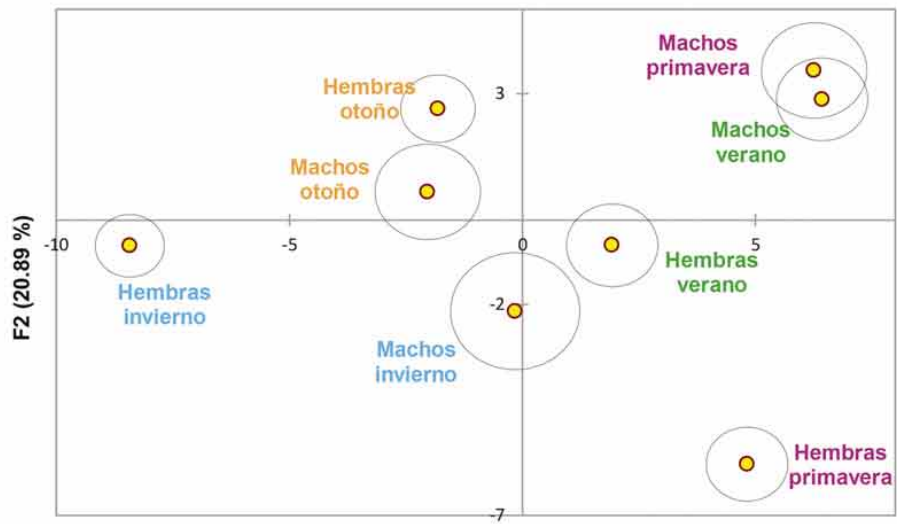

F1 (66.87\%)

Fig. 6. Diagramas del Análisis Discriminantes de Xochimilco A y B; y de Tecocomulco C y D. Considerando daños tisulares, LPO, índices somáticos (IHS, IGS y MCE), sexo y edad de F. americana durante un ciclo anual (primavera, verano, otoño e invierno). Daños tisulares: infiltración leucocitaria (IN), vasocongestión (VA), grados de daño I, II, III.

dos y más años) y con IN III. En el cuadrante superior derecho se localizan los machos y las hembras de otoño donde se relacionan con los mayores IHS y las combinaciones de daños histológicos de mayor grado (IN II-VA III e IN IIIVA III); y las hembras de invierno que se ubican junto al eje F1 presentaron lesiones hepáticas de IN III e IN II-VA I y corresponden a gallaretas menores de un año de edad.

El Análisis Discriminante de Tecocomulco (Figs. 6C y 6D), alcanzó el $87,76 \%$ de la varianza explicada en sus dos primeros componentes $(66,87 \%$ en el primer componente y $20,89 \%$ en el segundo componente). En el diagrama se observa que se separan las hembras y los machos de primavera y verano en el cuadrante derecho. Los machos en el cuadrante superior derecho se relacionan con la mayor MCE, son individuos de un año de edad, y con daños histológicos IN II, IN III e IN III-VA II. Las hembras en el cuadrante inferior derecho se asocian con el mayor IGS, son hembras mayores de dos años y con daños histológicos IN I-VA II y VA III. Las hembras y los machos de otoño se agrupan en el cuadrante superior izquierdo caracterizados por los IHS más altos y la presencia de daños histopatológicos IN I, VA II, IN III-VA III e IN II-VA II (Figs. 6C y 6D). Durante el invierno las hembras y los machos se ubican en el cuadrante inferior izquierdo. No obstante, las hembras se agrupan cerca del eje que separa los cuadrantes superior e inferior en relación con los niveles más altos de LPO, daños histológicos IN II-VA II y la edad (individuos de dos años y los menores de un año). Los machos de invierno se ubican en el eje que colinda con el cuadrante inferior derecho y se relacionan con VA I, IN II-VA III (Figs. 6C y 6D). 
LÓPEZ-ISLAS, M. E.; IBARRA-MEZA, I.; ORTIZ-ORDÓÑEZ, E.; FAVARI, L.; SEDEÑO-DÍAZ, J. E. \& LÓPEZ-LÓPEZ, E. Histopatología del hígado, lipoperoxidación e índices somáticos de Fulica americana en los humedales de Xochimilco (urbano) y Tecocomulco (rural), de la Cuenca de México. Int. J. Morphol., 34(2):522-532, 2016.

\section{DISCUSIÓN}

Los resultados de las características del agua indican que Xochimilco, presenta un mayor grado de eutrofización que Tecocomulco, debido a sus altas concentraciones de nutrientes (nitratos, nitritos y ortofosfatos) y sales (cloruros y sulfatos); lo anterior, es debido a que su abasto de agua proviene principalmente de plantas de tratamiento de aguas residuales (Ramsar); de las actividades agrícolas realizadas en las chinampas y de las descargas domésticas de la zona urbana (Abeja-Pineda et al., 2015). Los nitritos se encuentran en altas concentraciones en los cuerpos de agua en los que hay descargas de drenajes, tal es el caso de Xochimilco. Por su parte, Tecocomulco es un cuerpo de agua endorreico y fuertemente alcalino, en cuyos alrededores se realizan actividades agrícolas con el consecuente uso de agroquímicos (Ramsar). Aunado a lo anterior, está el arrastre de sedimentos de toda la cuenca que tiene un alto grado de deforestación, que afecta las condiciones fisicoquímicas del agua (Ramsar), como fue el caso del incremento de los SST, el color, el P total, ortofosfatos y $\mathrm{N}$ totalobservado en la época de lluvias, lo cual coincide con lo encontrado en Tecocomulco por De la Lanza \& Hernández- Pulido. La gran cantidad de coliformes fecales encontrados en Tecocomulco probablemente es debida a que en los alrededores forrajean ganado ovino y bovino (Ramsar).

Los valores del IGS indican que el período de reproducción tuvo lugar durante la primavera y el verano, lo que coincide con lo reportado para la especie (Brisbin et al.), además se observó que el periodo de reproducción fue mayor en Tecocomulco que en Xochimilco. Entre los mecanismos que subyacen al costo de la reproducción están: la regulación hormonal, el metabolismo intermediario, la distribución de los recursos, la función inmunológica, las proteínas para la reproducción, y las defensas contra el estrés y la toxicidad (Harshman \& Zera, 2006). Los factores que pudieron favorecer que en Tecocomulco se hayan alcanzado IGS mayores durante la primavera que en Xochimilco son los altos valores de los IHS y la MCE, lo que a su vez es resultado de que en Tecocomulco las gallaretas viven en condiciones de mejor calidad del agua; mientras que en Xochimilco, los bajos IGS están relacionados con los bajos valores de la MCE, el IHS, y una menor calidad del agua ya que en primavera (sequía cálida) se registraron los máximos valores de $\mathrm{P}$ total, ortofosfatos, nitritos y coliformes fecales. No obstante, en Tecocomulco, el costo de la reproducción en primavera se reflejó en una mayor LPO hepática, en relación con las gallaretas de Xochimilco que tuvieron la menor LPO hepática. Lo anterior sugiere que las gallaretas de Tecocomulco presentaron un balance de costo-beneficio con una mayor inversión de recursos para la reproducción y en consecuencia una menor respuesta de defensa antioxidante. El esfuerzo reproductivo ha sido reconocido como un proceso con un gasto energético muy elevado para las aves (Bannor, 1995), y también se considera un factor promotor de estrés oxidativo (Pamplona \& Costantini). Por el contrario, en Xochimilco, el balance costo-beneficio fue a favor de una mayor defensa antioxidante, no obstante, se vio reflejado con un periodo reproductivo más corto y postergado en comparación con el de Tecocomulco.

Los niveles de la LPO hepática encontrados en las gallaretas pueden explicarse en dos contextos: uno de acuerdo con la teoría de las historias de vida (Costantini, 2008), y el otro por los agentes pro-oxidantes. De acuerdo con la teoría de las historias de vida la presencia de depredadores puede ser un factor promotor del aumento del estrés oxidativo (Travers et al., 2010), al igual que la competencia, la calidad y cantidad de alimento, entre otros factores ambientales lo que explica los elevados niveles de LPO registrados por las gallaretas de Tecocomulco durante el invierno, que coinciden con la temporada de caza y con el arribo de las aves migratorias. Por su parte, los agentes pro-oxidantes exógenos (los contaminantes) también aumentan la producción intracelular de especies reactivas de oxígeno en el organismo causando daños celulares y tisulares que tienen un papel muy importante en la patofisiología de muchas enfermedades (Costantini). Al comparar los resultados de LPO obtenidos en F. americana (especie residente) en este trabajo con los obtenidos por Rajaaho et al. en Hirundo rustica, que es una especie migratoria, durante un ciclo anual, se encontró que en invierno ambas especies registraron los valores máximos de LPO hepática. Tanto en Tecocomulco como en Suráfrica llegan gran cantidad de aves migratorias durante el otoño e invierno, los resultados de los altos niveles de LPO en invierno, sugieren que en las áreas donde pasan el invierno las parvadas de especies migratorias, se ejerce estrés oxidativo tanto en las poblaciones residentes como en las migratorias.

El sistema inmunológico es uno de los principales componentes fisiológicos del auto-mantenimiento y promueve la supervivencia al reducir la probabilidad de mortalidad relacionada con enfermedades, lo cual implica costos energéticos, de manera que durante el ciclo anual, se pueden presentar balances costo-beneficio entre el sistema inmune y otras funciones del organismo (Hegemann et al., 2012). El proceso inflamatorio está íntimamente ligado a la respuesta inmune y a otros procesos de homeostasis como la regulación térmica, la circulación sanguínea, etc., y constituye la respuesta del organismo al daño causado por diversos agentes como traumatismos, virus, bacterias, parásitos entre otros (Bernet et al., 1999). La IN es una alteración del proceso inflamatorio que se observó en las gallaretas de Xochimilco y de Tecocomulco durante todo el ciclo anual, ya sea como daño 
único o combinado con la VA. La VA es una alteración circulatoria que resulta de una condición patológica del flujo sanguíneo y de los fluidos tisulares. Las hembras de Tecocomulco tuvieron una mayor prevalencia de VA principalmente en primavera e invierno. La relevancia de las lesiones depende de su importancia patológica, es decir, en cómo afecta la función del órgano y la capacidad del organismo para sobrevivir (Bernet et al.). Los resultados obtenidos muestran que las gallaretas presentaron cambios histopatológicos con diferentes grados de intensidad entre épocas del año, localidades y, sexos, lo cual sugiere que ambas poblaciones están sujetas a factores ambientales, químicos, físicos y biológicos que inducen estas respuestas. Según Bernet et al., la VA es una alteración patológica de mínima importancia que es fácilmente reversible cuando la exposición a los irritantes termina. La IN es una lesión de importancia patológica moderada que es reversible en la mayoría de los casos si el estresor es neutralizado. No obstante, en las dos localidades de estudio las gallaretas están expuestas de forma crónica a los diferentes estresores ya que la especie es residente de ambos cuerpos de agua lo que implicaría que los daños incrementen hasta alcanzar daños irreversibles afectando a la población. En particular, la mayor prevalencia de IN en Xochimilco, se puede atribuir a que este cuerpo de agua recibe aguas residuales continuamente (Abeja-Pineda et al.), lo que puede ser un factor de ingreso de patógenos. Binkowski et al. (2013) realizaron un estudio histopatológico en Anas platyrhynchos y Fulica atra de vida silvestre donde encontraron lesiones hepáticas como VA, IN, necrosis y hemosideresis, relacionadas con la presencia de plomo y cadmio, en Xochimilco y Tecocomulco se ha reportado la presencia de metales pesados (Abeja-Pineda et al.; Ramsar).

En ambas localidades $F$. americana enfrenta diversos estresores ambientales que afectan la salud de la especie durante todo el ciclo anual, la detección de daños tempranos que pueden ser reversibles puede permitir el evitar daños mayores si se realizan medidas de manejo para mitigar los efectos nocivos en las gallaretas, por ejemplo, el mejorar la calidad de agua de los humedales evitando la descarga de drenajes y reevaluar el efecto de la cacería en los humedales para adecuar las temporadas de caza.

Los resultados de las investigaciones en organismos de vida silvestre reflejan efectos de múltiples estresores, incluyendo todas las posibles interacciones entre los compuestos químicos tóxicos, los estresores ambientales, los recursos alimentarios disponibles y la interacción entre especies. Por lo que, los trabajos realizados en campo contribuyen a observar y monitorear los efectos de los eventos que se presentan en los ecosistemas, de tal manera que el uso combinado de biomarcadores y la evaluación histopatológica permite evaluar la salud de los organismos en vida silvestre.
En ambas localidades la reproducción es una época crítica, en la que cada población de gallareta la enfrenta de diferente manera: en Tecocomulco hay un balance costo beneficio a favor de la reproducción, con una combinación de los dos daños histológicos, mientras que en Xochimilco a pesar de que se presentó una menor LPO se observó una mayor IN, y el periodo reproductivo fue menor.

Los biomarcadores de daño temprano reflejan las etapas críticas de las dos poblaciones en el transcurso del año: la reproducción en primavera-verano, y adicionalmente en Tecocomulco se presenta otro momento de estrés por el arribo de aves migratorias y la cacería en el invierno.

F. americana es una especie que puede ser utilizada como centinela debido a que con el uso de biomarcadores histológicos y bioquímicos e índices somáticos se mostraron las respuestas diferenciadas en las distintas localidades, sexos y épocas del año.

\section{AGRADECIMIENTOS}

Los autores agradecen al Instituto Politécnico Nacional el financiamiento otorgado al presente trabajo a través de los proyectos SIP 20111126, 20110602 y 20120931 y a la Auxiliar de Investigación María Teresa García Camacho por el trabajo de laboratorio realizado. Así mismo agradecen a Carlos López Islas (CLI) por la edición de las figuras.

LÓPEZ-ISLAS, M. E.; IBARRA-MEZA, I.; ORTIZ-ORDÓÑEZ, E.; FAVARI, L.; SEDEÑO-DÍAZ, J. E. \& LÓPEZ-LÓPEZ, E. Liver histopatology, lipidperoxidation and somatic indices of Fulica americana in Xochimilco (urban) and Tecocomulco (rural) wetlands in the Mexico Basin. Int. J. Morphol., 34(2):522-532, 2016.

SUMMARY: Wetlands are very productive ecosystems and water birds complete their lifecycle there. Unfortunately, they constantly receive xenobiotics which can cause damage in aquatic organisms. This work assessed the health conditions of Fulica americana through histopathological and oxidative stress biomarkers and somatic indices in two wetlands from the Mexico Basin to evaluate the effect of habitat conditions on the birds health. The specimens were collected from Xochimilco (X) and Tecocomulco (T) wetlands during an annual cycle. They were eviscerated and weighed. Liver samples were taken for histological analysis and to quantify the lipid peroxidation (LPO) in the laboratory. The coots were aged. The water quality from both wetlands was analyzed. The liver tissue was embedded in liquid paraffin and was stained with hematoxylin and eosin. Two pathologies were detected in three levels each (I, II and III): leukocyte infiltration (IN) and vasocongestion (VA). The highest prevalence of both damages were observed in T coots, while the highest IN percentage was detected in X coots. The maximum LPO levels were detected during winter at $\mathrm{T}$, which could be related to hunter season and the wintering migratory birds. In X the highest LPO levels 
LÓPEZ-ISLAS, M. E.; IBARRA-MEZA, I.; ORTIZ-ORDÓÑEZ, E.; FAVARI, L.; SEDEÑO-DÍAZ, J. E. \& LÓPEZ-LÓPEZ, E. Histopatología del hígado, lipoperoxidación e índices somáticos de Fulica americana en los humedales de Xochimilco (urbano) y Tecocomulco (rural), de la Cuenca de México. Int. J. Morphol., 34(2):522-532, 2016.

were related to breeding season. The biggest eviscerated body mass was noted in $\mathrm{T}$ males and the smallest in the $\mathrm{X}$ females. All coots reached the highest hepatosomatic index during autumn and the minimum during spring. American coot can be used as sentinel because it is able to present differentiated responses, somatic indices, histopathological biomarkers and oxidative stress in different locations, sex and periods of the year.

KEY WORDS: Aquatic birds; Histological biomarkers; Oxidative stress; Ramsar wetlands; Seasonal variations.

\section{REFERENCIAS BIBLIOGRÁFICAS}

Abeja-Pineda, O.; López-López, E.; Favari, L. \& Sedeño-Díaz, J. E. Algal growth potential and oxidative stress in Ankistrodesmus falcatus exposed to waters from Xochimilco Lake system, México. Aquat. Ecosyst. Health Manag., 18(2):221-31, 2015.

American Public Health Association (APHA). Standard Methods: for the Examination of Water and Wastewater. $16^{\text {th }}$ ed. Washington D. C., APHA-AWWA-WEF, 2005.

Bannor, B. K. Continuous breeding by American coots in Dade County, Florida. Fla. Field Nat., 23(4):87-92, 1995.

Bernet, D.; Schmidt, H.; Meier, W.; Burkhardt-Holm, P. \& Wahli, T. Histopathology in fish: proposal for a protocol to assess aquatic pollution. J. Fish Dis., 22(1):25-34, 1999.

Binkowski, L. J.; Sawicka-Kapusta, K.; Szarek, J.; Strzyzewska, E. \& Felsmann, M. Histopathology of liver and kidneys of wild living Mallards Anas platyrhynchos and Coots Fulica atra with considerable concentrations of lead and cadmium. Sci. Total Environ., 450-1:32633, 2013.

Bradford, M. M. A rapid and sensitive method for the quantitation of microgram quantities of protein utilizing the principle of protein-dye binding. Anal. Biochem., 72:248-54, 1976.

Brisbin, I. L. Jr.; Lehr, I. \& Mowbray, T. B. American Coot (Fulica americana). En: Poole, A. (Ed.). The Birds of North America Online. Ithaca, Cornell Lab of Ornithology, 2002. Disponible en: http:// bna.birds.cornell.edu/bna/species/697a

Buege, J. A. \& Aust, S. D. Microsomal lipid peroxidation. Methods Enzymol., 52:302-10, 1978.

Costantini, D. Oxidative stress in ecology and evolution: lessons from avian studies. Ecol. Lett., 11(11):1238-51, 2008.

De la Lanza, E. G. \& Hernández Pulido, S. Fisicoquímica de la Laguna de Tecocomulco. En: Huízar-Álvarez, R.; Jiménez-Fernández, E. J. \& Juárez-López, C. (Eds.). La Laguna de Tecocomulco. Geo-Ecología de un Desastre. México D. F., Publicación Especial 3, Instituto de Geología, Universidad Nacional Autónoma de México, 2005. pp.10928 .

Harshman, L. G. \& Zera, A. J. The cost of reproduction: the devil in the details. Trends Ecol. Evol., 22(2):80-6, 2006.

Hegemann, A.; Matson, K. D.; Both, C. \& Tieleman, B. I. Immune function in a free-living bird varies over the annual cycle, but seasonal patterns differ between years. Oecologia, 170(3):605-18, 2012.
Koivula, M. J. \& Eeva, T. Metal-related oxidative stress in birds. Environ. Pollut., 158(7):2359-70, 2010.

Labocha, M. K. \& Hayes, J. P. Morphometric indices of body condition in birds: a review. J. Ornithol., 153(1):1-22, 2012.

Malik, K.; Lone, K. P.; Khansa; Rashid, F.; Fakhar-un-Nisa; Naz, S.; Sharif, S. \& Awan, K. Effect of feeding rapeseed meal on the liver weight and hepato-somatic index (HIS) content of liver of Japanese quail. Afr. J. Microbiol. Res., 6(9):1918-23, 2012.

Pamplona, R. \& Costantini, D. Molecular and structural antioxidant defenses against oxidative stress in animals. Am. J. Physiol. Regul. Integr. Comp. Physiol., 301(4):R843-63, 2011.

Pérez-Arteaga, A. \& Gaston, K. J. Status of American Coot Fulica americana (Gruiformes: Rallidae) wintering in Mexico. Acta Zool. Mex., 20(1):253-63, 2004.

Pyle, P. Identification Guide to North American Birds. Part II: Anatidae to Alcidae. California, Slate Creek Press, 2008.

Raja-aho, S.; Kanerva, M.; Eeva, T.; Lehikoinen, E.; Suorsa, P.; Gao, K.; Vosloo, D. \& Nikinmaa, M. Seasonal variation in the regulation of redox state and some biotransformation enzyme activities in the barn swallow (Hirundo rustica L.). Physiol. Biochem. Zool., 85(2):148$58,2012$.

Ramsar. Fichas Informativas de Ramsar. Gland, Ramsar, 2015. Disponible en: http://www.ramsar.org/es/recursos/fichas-informativas-deramsar

Travers, M.; Clinchy, M.; Zanette, L.; Boonstra, R. \& Williams, T. D. Indirect predator effects on clutch size and the cost of egg production. Ecol. Lett., 13(8):980-8, 2010.

Uría, E. \& Mora, C. Apuntes para cl Curso Teórico Práctico de Histología Animal. México D. F., ENCB, IPN, 1996.

van der Oost, R.; Beyer, J. \& Vermeulen, N. P. Fish bioaccumulation and biomarkers in environmental risk assessment: a review. Environ. Toxicol. Pharmacol., 13(2):57-149, 2003.

Wikelski, M.; Martin, L. B.; Scheuerlein, A.; Robinson, M. T.; Robinson, N. D.; Helm, B.; Hau, M. \& Gwinner, E. Avian circannual clocks: adaptive significance and possible involvement of energy turnover in their proximate control. Philos. Trans. R. Soc. Lond. B Biol. Sci., 363(1490):411-23, 2008

Williams, T. D. Physiological Adaptations for Breeding in Birds. New Jersey, Princeton University Press, 2012.

\section{Dirección para Correspondencia:}

Dra. Eugenia López

Laboratorio de Evaluación de la Salud de los Ecosistemas Acuáticos

Escuela Nacional de Ciencias Biológicas - IPN (ENCB-IPN)

Prol. de Carpio y Plan de Ayala s/n, Col. Santo Tomás

CP 011340

México D.F. - MÉXICO

Email: eugenia_lopez@hotmail.com Recibido : 08-11-2015 Aceptado: 22-01-2016 